\title{
DRY FRICTION MODEL OF A CONTACT OF A SOLID BALL MOVING ALONG THE BOUNDARIES OF TWO RAILS
}

\author{
ALEXEY A. KIREENKOV ${ }^{1,2}$ \\ ${ }^{1}$ Moscow Institute of Physics and Technology (State University), Institutskiy per. 9, Dolgoprudny, \\ Moscow Region, 141701, Russia, kireenkov.aa@mipt.ru \\ ${ }^{2}$ Institute for Problems in Mechanics of Russian Academy of Sciences, 119526, Prospect \\ Vernadskogo 101, korp.1, Moscow, Russia, kireenk@ipmnet.ru, kireenk@mail.ru
}

Key words: Combined Dry Friction, "Butterfly" Robot.

\begin{abstract}
It is proposed applications of the theory of combined dry friction [1-14] in the problem of the ball rolling on boundaries of two identical frames. Father development of this model can be used in investigation of the dynamic of the a so-called "Butterfly" robot, consisting of two identical shaped plates rigidly placed parallel to each other on a small distance aimed at manipulating a ball that can freely roll on the plates' boundaries as on rails [15]. The difficult control system, considered in [16-18] are another engineering application of the combined dry theory. Following developed in previous investigation approach, the friction force and torque are computed by the integration over the contact area so that the exact dynamically coupled integral model accounting the relationship of all the components of friction is obtained. This exact model is replaced by approximated analytical model [6] which is completely satisfy to all analytical properties of integral model as function kinematics parameters without increasing the number of coefficients.
\end{abstract}

\section{INTRODUCTION}

The problem of the ball rolling on boundaries of two identical frames is interested applications of the theory of the combined dry friction. It was on the example of the study of the classical problem about the dynamics of a ball on a rough plane that the necessity of creating this theory and its further development were demonstrated. The distinguishing feature of this theory is presenting a more convenient form of the coupled friction models for the problems of solids dynamics. One of the main assumptions of this theory is the validity of the classical Amonton-Coulomb law in differential form for a small element of the area inside the contact spot of the rubbing solids. Then, the net vector of the friction force and torque are computed by the integration over the contact area and presents the exact coupled integral models of the combined dry friction. To avoid calculating double integrals, the exact model is replaced by an approximated analytical model [1-14], which fully satisfies all the analytical properties of the integral model as a function of kinematic parameters without increasing the number of coefficients.

Moreover, the friction model of ball rolling friction along two rigidly connected rails is not only of independent interest. This model can also be used in the future in order to describe 
more correctly the effects observed under investigation of the dynamics of the so-called "Butterfly" robot [15]. "Butterfly" robot consists from the two identical shaped plates rigidly placed parallel to each other on a small distance aimed at manipulating a ball that can freely roll on the plates' boundaries as on rails.

\section{PROBLEM STATEMENT AND BASIC ASSUMPTIONS}

A free rolling of a heavy uniform ball of radius $R_{b}$ (Fig.1.b) along the two identical rails rigidly placed parallel to each other (Fig.1.a) is considered (Fig.1). It is supposed that the ball is involved in the complex motion: simultaneous rolling, spinning and sliding along the rails.

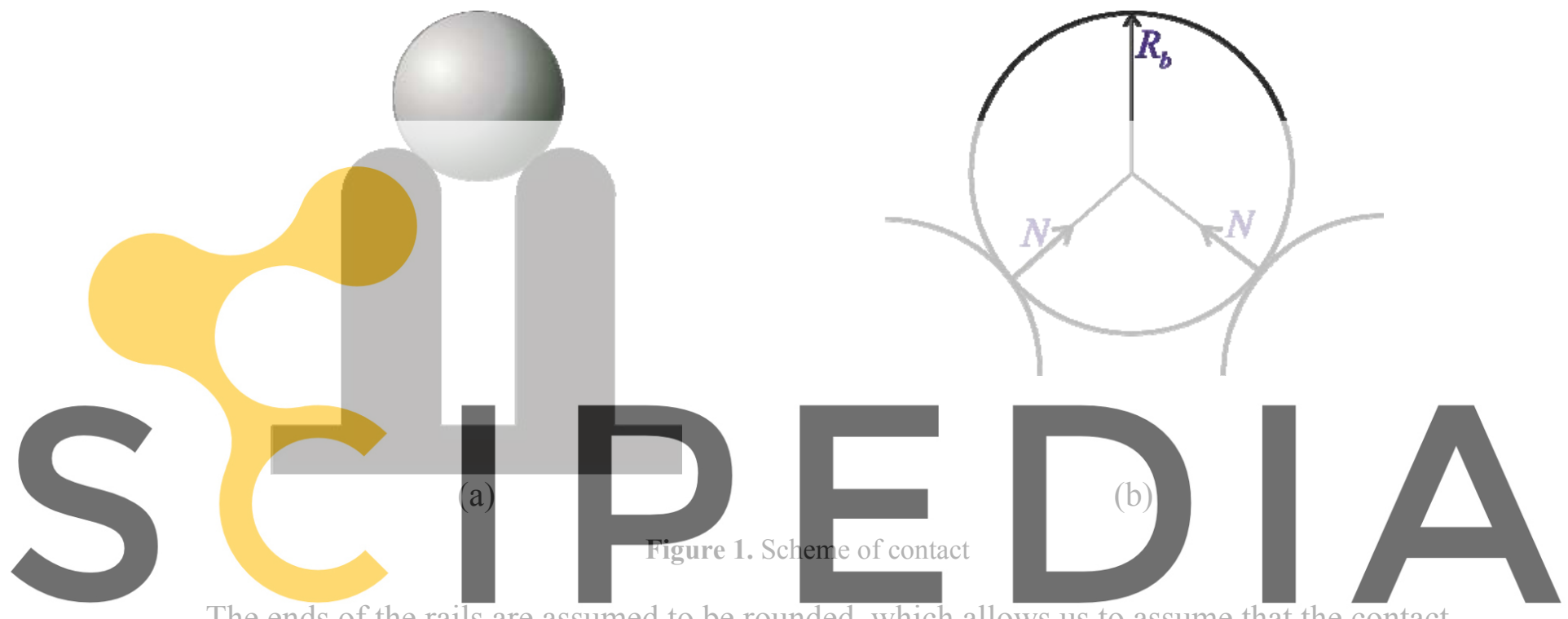

The ends of the rails are assumed to be rounded, which allows us to assume that the contact Register furf fres of the rubbing bodies are locally spherical and the contact. spots have circle form. Under these assumptions, the distributions of the normal contact stresses inside of contact spots at the absence of ball motion are can be described by the Hertz law [19-20]:

$$
\sigma_{i 0}(x, y)=\frac{3 N}{2 \pi R^{2}} \sqrt{1-\frac{x^{2}}{R^{2}}-\frac{y^{2}}{R^{2}}}, i=1,2
$$

Here index $i$ denotes the number of contact spot, but $x$ and $y$ are coordinates of the small areas inside of contact spots (Fig.2.a) in the rectangular systems of coordinates $\left\{O_{i} e_{i 1} e_{i 2} e_{i 3}\right\}, i=1,2$. Origins of the considered coordinates systems are hardly connected with the centers of contact circles. Unit vectors $e_{i 1}$ are directed along the direction of the ball sliding, parallel to the rails. Unit vectors $e_{i 3}$ are directed perpendicularly to the direction of the ball sliding and complement vectors $e_{i 1}$ and $e_{i 3}$ up to the right triple.

Another main assumption is that the Amonton-Coulomb law in differential form holds for the small surface element $d S_{i}$ in the interior of the contact spot. According to this law, the 
differentials of the resultant vector $d \mathbf{F}_{i}$ and the moment of friction $d M_{i C}$ with respect to the contact spot center are determined by the following formulae [1-14]:

$$
d \mathbf{F}=-f \sigma \frac{\mathbf{V}}{|\mathbf{V}|} d S, d M_{C}=-f \sigma \frac{\mathbf{r} \times \mathbf{V}}{|\mathbf{V}|} d S, \mathbf{V}=(v-\omega y, \omega x), \mathbf{r}=(x, y)
$$

where $f$ is the coefficient of friction; $\mathbf{r}=(x, y)$ is the position vector of an elemental area in the interior of the contact spot with respect to its center Fig. 2.a; $v$ is velocity of the relative sliding inside of contact spot, but $\omega$ is the component of the angular velocity of the ball rotation respectively to the contact spot plane. The velocity $\omega$ can be considered as velocity of the contact spot rotation relatively to the ball. It's perpendicular directed to the contact spot in the direction the ball radius. In addition, index $i$ in formulas (2) is omitted for briefly of writing.

\section{DRY FRICTION MODEL}

There are well known results from the theory of elasticity [15] that tangent stresses lead to shift in the symmetric diagram of the normal contact stresses in the direction of the instantaneous sliding velocity $v$ or in the rolling direction Fig2.b.

To use these results in the dynamics problems, it is proposed the simple asymptotic representations for the contact stresses distributions based on their general properties [1-14]:
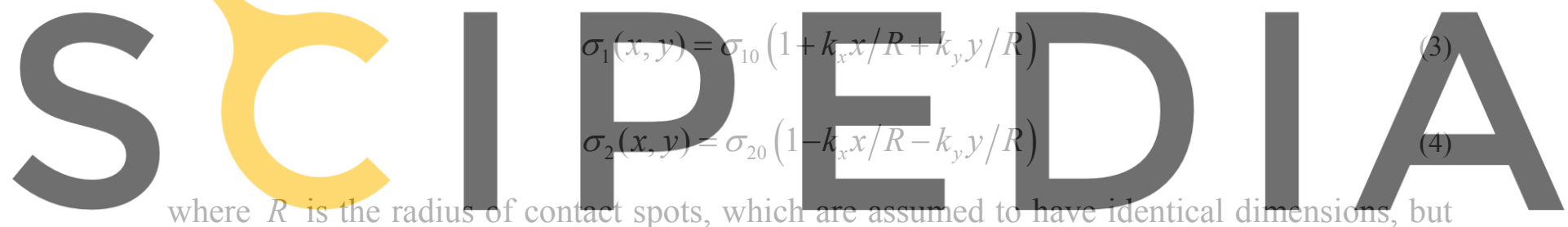

$k_{x}$ and $k_{v}$ are dimensionless coefficients that describe the components of the displacement of

Register for free at https//www.scipedia.com to download the version without the watermark the center of gravity of the contact spot relative to its geometric center.

The difference in signs in formulas (3)-(4) is connected with difference in direction of the ball rotation relative to the contact spots.

Presence of the simultaneously sliding and rolling in the different directions leads only to summarization of the corresponded coefficients, which can be used on the base of the results of theory of elasticity [15] or procedure developed in [1-14].

The displacement of the contact spot gravity results to the appearance of the rolling moment $\mathbf{M}_{\mathrm{r}}^{i}$ parallel to the contact plane whose projections parallel $\left(M_{\|}\right)$and perpendicular $\left(M_{\perp}\right)$ to direction of the relative sliding can be determined by the formulas from [1-14].

$$
\mathbf{M}_{\mathbf{r}}^{\mathbf{i}}=M_{\|}^{i} \mathbf{e}_{1}^{\mathbf{i}}+M_{\perp}^{i} \mathbf{e}_{2}^{\mathbf{i}}, i=1,2
$$




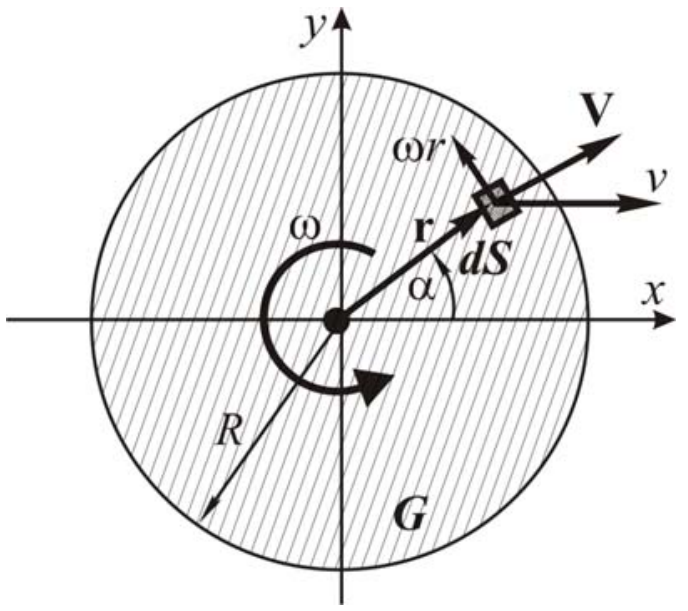

(a)

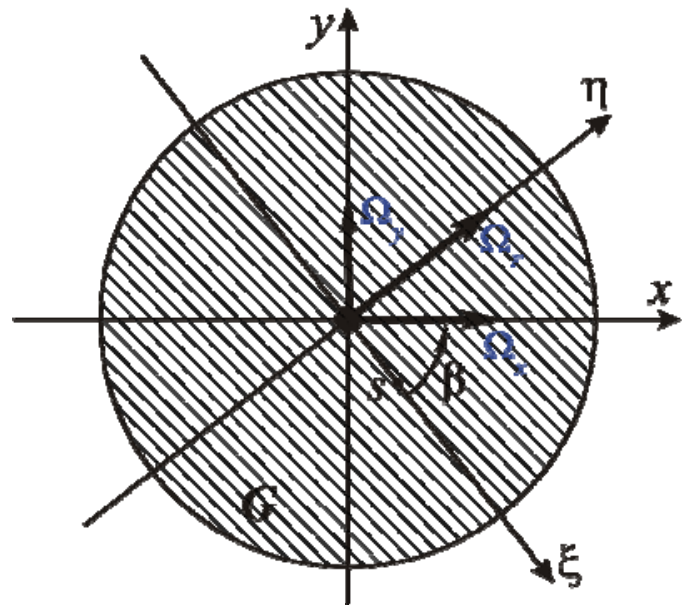

(b)

Figure 2. Kinematics inside the contact spot.

Integration of the corresponded differentials over the contact spot yields the resultant vector $\mathbf{F}$ of the friction force and torque $\mathbf{M}_{\mathbf{C}}$ :

$$
\mathbb{F}=-f \iint_{G} \sigma(x, y) \frac{\mathbf{V}}{|\mathbf{V}|} d x d y, \quad \mathbf{M}_{\mathbf{C}}=-f \iint \sigma(x, y) \frac{\mathbf{r} \times \mathbf{V}}{|\mathbf{V}|} d x d y
$$
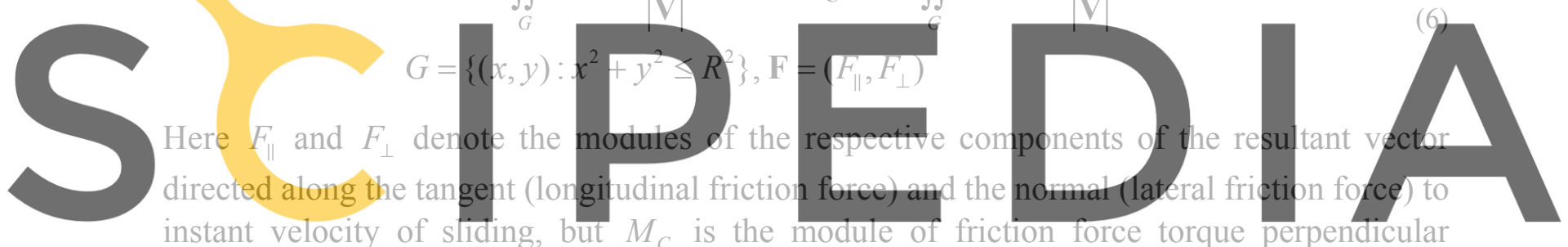

Register for free at hteps/fwhw. scipe:dia.com to download the version without the watermark

$$
\mathbb{F}=F_{\|} \mathbf{e}_{1}+F_{\perp} e_{2}, \mathbf{M}_{\mathrm{C}}=M_{C} \mathbf{e}_{3}
$$

The exact integral models gives a good description of the dry friction effects in the case of combined kinematics, but is inconvenient to be used in problems of dynamics, because it is required to calculate multiple integrals in the right-hand sides of the equations of motion.

Approximated model [1-14] based on the analytical properties of the integral model as a function of kinematic parameters allowed to avoid this inconvenience without increasing the number of coefficients. Coefficients of this model are numbers that can be easy calculated [114] or identified from experiments [21-22] in more complicated cases. This fact permits use results of previous investigation directly in considered problem. Thus, the dry friction model which is described the interaction between ball and rails can be represented in following form:

$$
\begin{aligned}
& M_{C}=\frac{M_{0} u}{\sqrt{u^{2}+m v^{2}}}, F_{\|}=\frac{F_{0} v}{\sqrt{v^{2}+a u^{2}}}, F_{\perp}=\frac{\mu F_{0} u}{\sqrt{\left(u^{2}+b v^{2}\right)}} \\
& M_{\|}=-M_{r} \frac{\Omega_{y}}{\Omega_{r}}, M_{\perp}=-M_{r} \frac{\Omega_{x}}{\Omega_{r}}, M_{r} \equiv s k_{r} N
\end{aligned}
$$


Coefficients of this model were calculated in previous works [1-14], but, as above, index $i$ is omitted for briefly of writing.

\section{ACKNOWLEDGEMENTS}

These investigations were supported by the RFBR, grant 20-08-01120 and partly by the state program of the scientific research works on the section No.: AAAA-A20120011690138-6.

\section{REFERENCES}

[1] Kireenkov, A.A. Improved theory of the combined dry friction in problems of aviation pneumatics dynamics. Proceedings of the 7th International Conference on Coupled Problems in Science and Engineering, COUPLED PROBLEMS 2017 (2017):1293-1298.

[2] Kireenkov, A.A. Improved friction model of the aviation tyre contact with the landing strip. IFAC-PapersOnLine (2018) 51(2) :890-894. (DOI 10.1016/j.ifacol.2018.04.027)

[3] Kireenkov, A.A., Nushtaev, D.V., Zhavoronok, S.I. A new approximate model of tyre accounting for both deformed state and dry friction forces in the contact spot on the background of the coupled model. MATEC Web of Conferences (2018) 211(08003). (DOI 10.1051/matecconf/201821108003)

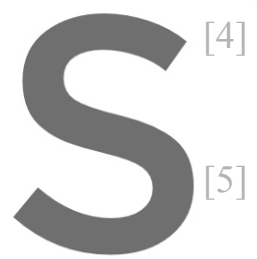

4] Kireenkov, A.A., Zhavoronok, S.I. Coupl pneumatics dynamics. International Jou 203. (DOI 10.1016/j.ijmecsci.2017. Kireenkov, A.A., Zhavoronok, S.I. Implen
combined dry friction in problems
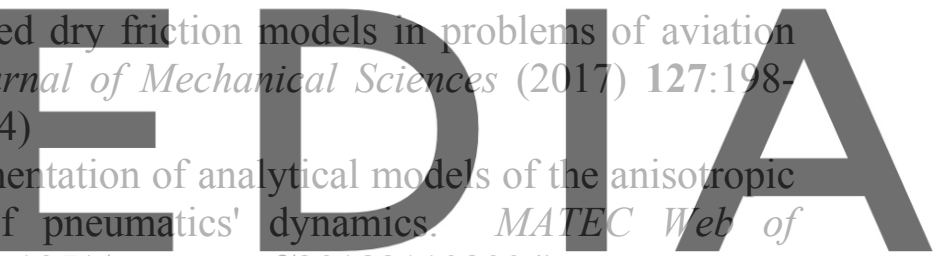
Conferences (2018) 211(08004). (DOI 10.1051/matecconf/201821108004)

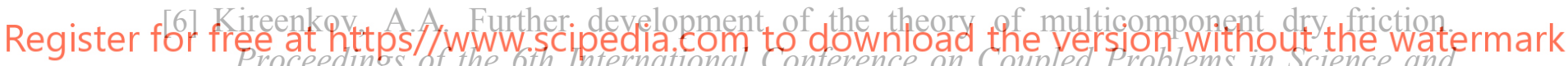
Engineering, COUPLED PROBLEMS 2015 (2015):203-209.

[7] Kireenkov, A.A. Three-dimensional model of combined dry friction and its application in non-holonomic mechanics. Proceedings of ENOC-2005, Eindhoven, Netherlands, August 7-12 (2005):571-577.

[8] Ramodanov, S.M., Kireenkov, A.A. Controllability of a rigid body in a perfect fluid in the presence of friction. Proceedings of the 7th International Conference on Coupled Problems in Science and Engineering, COUPLED PROBLEMS 2017 (2017):179 - 184.

[9] Zhavoronok, S.I., Kireenkov, A.A. On the effect of the anisotropic dry friction and the deformed state of tires on the shimmy initiation. Proceedings of the 7th International Conference on Coupled Problems in Science and Engineering, COUPLED PROBLEMS 2017 (2017):216-226.

[10] Kireenkov, A.A., Zhavoronok, S.I. Anisotropic Combined Dry Friction in Problems of Pneumatics' Dynamics, Journal of Vibrational Engineering and Technologies (2020) 8 (2):365-372. (DOI: 10.1007/s42417-019-00140-1).

[11] Kireenkov, A.A., Ramodanov, S.M. Combined Dry Friction Models in the Case of Random Distribution of the Normal Contact Stresses Inside of Contact Patches, 
Proceedings of the 8th International Conference on Coupled Problems in Science and Engineering, COUPLED PROBLEMS 2019 (2019):176-182.

[12] Zhavoronok, S.I. The generalized Lagrange equations of the second kind for the extended three-dimensional $n$ 'th order theory of anisotropic shells. The J. of Mekhanika kompozitsionnykh materialov i konstruktsii (2015) 21(3):370-381.

[13] Kireenkov, A.A., Zhavoronok S.I. Numeric-Analytical Methods of the Coefficients Definition of the Rolling Friction Model of the Pneumatic Aviation Tire, Proceedings of the 8th International Conference on Coupled Problems in Science and Engineering, COUPLED PROBLEMS 2019 (2019):204-212.

[14] Kireenkov, A.A., Zhavoronok, S.I., Nushtaev, D.V. On tire models accounting for both deformed state and coupled dry friction in a contact spot. Computer Research and Modeling (2021) 13(1):163-173 (DOI: 10.20537/2076-7633-2021-13-1-163-173).

[15] Surov, M., Shiriaev, A. and ets., "Case study in non-prehensile manipulation: Planning and orbital stabilization of one-directional rollings for the 'Butterfly robot'", Proceedings - IEEE International Conference on Robotics and Automation (2015):14841489 .

[16] Semendyaev, S.V. Solid system with two massive eccentrics on a rough plane: rotational case. IFAC-Papers OnLine (2018) 51(2):884-889.

[17] Semendyaev, S.V. Coupled dynamics of solid system with slider-crank mechanisms as internal movers on rough surface with friction. Proceedings of the VII International

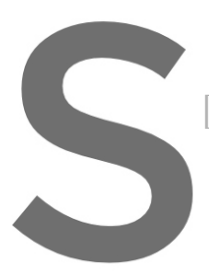
Conference on Coupled Problems in Science and Engineering (COUPLED PROBLEMS
2017) (2017):185-196 (ISBN: 978-84-943928-3).
Semendyaev, S.V. Gendral case of movement of solid systen with two massive
eccentrics on a rough planc. Proceeding of the VIl International Conference on
Coupled Problems in Sclence and Engineering (COUPLED PROBLEMS
2019) (2019):18B-191 (ISBN:978-84-949194-5-9).

[19] Kireenkov, A.A. Coupled models of sliding and rolling friction, Doklady Akademii Nauk

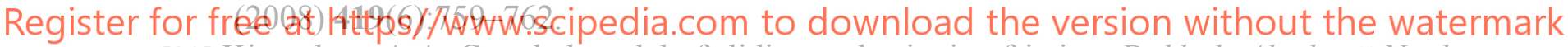

[20] Kireenkov, A.A. Coupled model of sliding and spinning friction, Doklady Akademii Nauk (2011) 441(6):750-755.

[21] Kireenkov, A.A., Semendyaev, S.V., Filatov, V.V. Experimental Study of Coupled Two-Dimensional Models of Sliding and Spinning Friction. Izv. Akad. Nauk. Mekh. Tverd. Tela. (2010) 6:192-202

[22] Kireenkov, A.A. Theoretical and experimental modelling of the Combined Dry Friction Effects. IFAC - MATHMOD - 2012 Proceedings, www.IFAC-PapersOnLine.net. 